\title{
FUNCTIONALS OF FINITE DEGREE AND THE CONVERGENCE OF THEIR FOURIER-HERMITE DEVELOPMENTS
}

\author{
ROSS E. GRAVES
}

1. Introduction. In this paper we define a class of functionals on the Wiener space $C$ which play in the theory of functionals on this infinite-dimensional space a role quite analogous to that of the polynomials on a space of finite dimension. These functionals we call functionals of finite degree; in particular we define functionals of degree $\boldsymbol{n}$. As in the finite-dimensional case we first define a functional to be of degree $n$ with respect to a fixed orthogonal coordinate system, and then show that the property of being a functional of degree $n$ is invariant under orthogonal transformation of coordinates. After a preliminary investigation of functionals of finite degree we establish the principal result of the paper, to the effect that the FourierHermite expansion of such a functional converges almost everywhere on $C$. This result is of interest in connection with the theory of multiple stochastic integrals developed by $R$. H. Cameron and the author, to appear in a separate paper.

Throughout this paper we use $\|f\|$ to mean the Hilbert $\left(L_{2}\right)$ norm of $f$. This convention applies both to functions defined on $[0,1]$ and to functionals defined on $C$.

In the work to follow we shall have occasion to use the generalization of the Riemann-Stieltjes integral due to Paley, Wiener, and Zygmund [3]. This integral, written $\int_{0}^{1} f(t) \bar{d} x(t)$, has the advantage that it is defined for almost all $x \in C$ for each $f \in L_{2}$ on $[0,1]$. If $f(t)$ is of $\mathrm{B}$. V., this generalized integral agrees with the ordinary Riemann-Stieltjes integral almost everywhere on $C$. If $f(t)$ is real and of class $L_{2}$ on $[0,1]$ and if $F(u)$ is Lebesgue measurable on $(-\infty, \infty)$, then

$$
\int_{C}^{w} F\left[\int_{0}^{1} f(t) \tilde{d} x(t)\right] d_{w} x=\pi^{-1 / 2} \int_{-\infty}^{\infty} F[\|f\| u] e^{-u^{2}} d u,
$$

where the existence of either side of (1.1) as an absolutely convergent integral implies that of the other and the equality. For the explicit definition of the generalized integral, and for the proofs of the above assertions and other properties of the generalized integral to be used in the sequel, we refer the reader to the original paper of Paley, Wiener, and Zygmund.

Received by the editors April 24, 1952. 
Definition 1. Let

$$
\begin{aligned}
\Psi_{m_{1}}, \cdots, m_{N} & (x)=\prod_{k=1}^{N} H_{m_{k}}\left[\int_{0}^{1} \alpha_{k}(t) \tilde{d} x(t)\right] \\
& \left(N=1,2, \cdots ; m_{k}=0,1,2, \cdots\right),
\end{aligned}
$$

where the $H_{m}$ 's are the partially normalized Hermite polynomials defined by

$$
H_{m}(u)=(-1)^{m} 2^{-m / 2}(m !)^{-1 / 2} e^{u^{2}} \frac{d^{m}}{d u^{m}}\left(e^{-u^{2}}\right) \quad(m=0,1,2, \cdots),
$$

and where $\left\{\alpha_{k}(t)\right\}$ is a C. O. N. (complete orthonormal) set (in the $L_{2}$ sense) of real-valued functions on $[0,1]$. Then the set $\left\{\Psi_{m_{1}}, \cdots, m_{N}(x)\right\}$ will be called the set of Fourier-Hermite polynomials (or functionals). We note that

$$
\Psi_{m_{1}}, \cdots, m_{N}(x) \equiv \Psi_{m_{1}}, \cdots, m_{N}, 0, \cdots, \theta(x), \quad \Psi_{0}(x) \equiv 1 .
$$

It has been shown by Cameron and Martin [1] that the FourierHermite functionals form a closed (in the $L_{2}(C)$ sense) orthonormal set of functionals. More explicitly, if for $F(x) \in L_{2}(C)$ we define

$$
A_{m_{1}, \cdots, m_{N}}=\int_{C}^{w} F(x) \Psi_{m_{1}, \cdots, m_{N}}(x) d_{w} x
$$

then

$$
\lim _{N \rightarrow \infty} \int_{C}^{w}\left|F(x)-\sum_{m_{1}, \cdots, m_{N}=0}^{N} A_{m_{1}, \cdots, m_{N}} \Psi_{m_{1}}, \cdots, m_{N}(x)\right|^{2} d_{w} x=0,
$$

and the Parseval equation holds in the sense that

$$
\lim _{N \rightarrow \infty} \sum_{m_{1}, \cdots, m_{N}=0}^{N}\left|A_{m_{1}}, \cdots, m_{N}\right|^{2}=\int_{C}^{w}|F(x)|^{2} d_{w} x .
$$

If we wish to call attention to the C. O. N. set $\left\{\alpha_{k}(t)\right\}$ used in the definition of the F. H. polynomials, we write $\Psi_{m_{1}}^{(\alpha)}, \ldots, m_{N}(x)$ and $A_{m_{1}, \cdots, m_{N}}$ in place of $\Psi_{m_{1}}, \cdots, m_{N}(x)$ and $A_{m_{1}, \cdots, m_{N}}$.

Definition 2. The degree of the F. H. polynomial $\Psi_{m_{1}}^{(\alpha)}, \cdots, m_{N}(x)$ is the non-negative integer $m_{1}+\cdots+m_{N}$.

Definition 3. A functional $F(x)$ on $C$ is said to be of degree $n$ with respect to the (real) C. O. N. set $\left\{\alpha_{k}(t)\right\}$ if it is of class $L_{2}(C)$ and if its F. H. development in terms of the functionals determined by $\left\{\alpha_{k}(t)\right\}$ contains no terms of degree greater than $n$ and at least one term of degree $n$. As a matter of convenience, a functional which is 
zero almost everywhere is assigned the degree -1 .

2. Invariance of the degree of a functional. We require a preliminary lemma.

Lemma 1. If $f(t) \in L_{2}$ on $[0,1]$, if $c_{j}=\int_{0}^{1} f(t) \alpha_{j}(t) d t$ where $\left\{\alpha_{j}(t)\right\}$ is a real $C . O$. $N$. set, and if $p$ is any positive number, we have

$$
\lim _{\nu \rightarrow \infty} \int_{C}^{w}\left|\int_{0}^{1} f(t) \tilde{d} x(t)-\sum_{j=1}^{\nu} c_{j} \int_{0}^{1} \alpha_{j}(t) \tilde{d} x(t)\right|^{p} d_{w} x=0 ;
$$

that is, $\sum_{j=1}^{v} c_{j} \int_{0}^{1} \alpha_{j}(t) \tilde{d} x(t)$ converges to $\int_{0}^{1} f(t) \tilde{d} x(t)$ in the $L_{p}(C)$ mean for every positive index $p$.

Proof. Without loss of generality we may suppose that $f(t)$ is real-valued. Upon setting $f_{\nu}(t)=\sum_{j-1}^{v} c_{j} \alpha_{j}(t)$, we have from (1.1)

$$
\begin{aligned}
\int_{C}^{w}\left|\int_{0}^{1} f(t) \tilde{d} x(t)-\sum_{j=1}^{\nu} c_{j} \int_{0}^{1} \alpha_{j}(t) \tilde{d} x(t)\right|^{p} d_{w} x & \\
& =\left\|f-f_{\nu}\right\|{ }^{p} \pi^{-1 / 2} \int_{-\infty}^{\infty}|u| p e^{-u^{2}} d u .
\end{aligned}
$$

As $\left\{\alpha_{j}(t)\right\}$ is closed, $\left\|f-f_{\nu}\right\| \rightarrow 0$, and the result follows.

We are now in a position to prove the invariance theorem.

TheOREM 1. If $F(x)$ is of degree $n$ with respect to a $C$. O. N. set $\left\{\alpha_{k}(t)\right\}$, then it is of degree $n$ with respect to any real C. O. N. set.

Proof. Let $\left\{\beta_{k}\right\}$ be any real C. O. N. set and for brevity write $b_{k}(x)=\int_{0}^{1} \beta_{k}(t) \tilde{d} x(t)$. With the notation of the introduction, by mean convergence and the fact that $F(x)$ is of degree $n$ with respect to $\left\{\alpha_{k}\right\}$ we find that

$$
\begin{aligned}
A_{m_{1}, \cdots, m_{N}}^{(\beta)} & =\int_{C}^{w} F(x) \Psi_{m_{1}, \cdots, m_{N}}^{(\beta)}(x) d_{w} x \\
= & \lim _{M \rightarrow \infty} \sum_{\mu_{1}+\cdots+\mu_{M} \leqq n} A_{\mu_{1}, \cdots, \mu_{M}}^{(\alpha)} \int_{C}^{w} \Psi_{\mu_{1}, \cdots, \mu_{M}}^{(\alpha)}(x) \\
& \cdot \Psi_{m_{1}, \cdots, m_{N}}^{(\beta)}(x) d_{w} x .
\end{aligned}
$$

Each integral $\int_{C}^{n} \Psi_{\mu_{1}}^{(\alpha)}, \ldots, \mu_{M}(x) \Psi_{m_{1}}^{(\beta)}, \ldots, m_{N}(x) d_{w} x$ is a finite sum of terms of the form

$$
\int_{c}^{w}\left\{\prod_{j=1}^{q} \int_{0}^{1^{1}} f_{j}(t) \tilde{d} x(t)\right\} \Psi_{m_{1}}^{(\beta)} \cdots, m_{N}(x) d_{w} x,
$$

where $f_{j} \in L_{2}$ on $[0,1]$ and where $q \leqq n$. From Lemma 1 and the properties of mean convergence we may conclude that 


$$
\begin{aligned}
\int_{C}^{w}\left\{\prod_{j=1}^{q} \int_{0}^{1} f_{j}(t) \tilde{d} x(t)\right\} \Psi_{m_{1}}^{(\beta)} \cdots, m_{N}(x) d_{w} x \\
=\lim _{\nu \rightarrow \infty} \int_{C}^{w}\left\{\prod_{j=1}^{q}\left[\sum_{k=1}^{\nu} c_{j k} b_{k}(x)\right]\right\} \Psi_{m_{1}, \cdots, m_{N}}^{(\beta)}(x) d_{w} x,
\end{aligned}
$$

where $c_{j k}=\int_{a}^{1} f_{j}(t) \beta_{k}(t) d t$. It is readily seen that $\prod_{j=1}^{g}\left[\sum_{t=1}^{k} c_{j k} b_{k}(x)\right]$ is expressible in the form

$$
\prod_{j=1}^{q}\left[\sum_{k=1}^{p} c_{j k} b_{k}(x)\right]=\sum_{r=1}^{R} C_{r} G_{r}(x),
$$

where the $G_{r}(x)$ 's are Fourier-Hermite functionals $\Psi_{i_{1}}^{(\beta)}, \ldots, i_{L}(x)$ of degree not greater than $q$. Thus if $m_{1}+\cdots+m_{N}>n$, we must have

$$
\int_{C}^{w} G_{r}(x) \Psi_{m_{1}, \cdots, m_{N}}^{(\beta)}(x) d_{w} x=0 .
$$

Combination of (2.1), (2.2), (2.3), and (2.4) shows that

$$
A_{m_{1}, \cdots, m_{N}}^{(\beta)}=0 \quad\left(m_{1}+\cdots+m_{N}>n\right) .
$$

From (2.5) we conclude at once that $F(x)$ is a functional of degree $m \leqq n$ with respect to $\left\{\beta_{k}\right\}$. If we now interchange the roles of $\left\{\alpha_{k}\right\}$ and $\left\{\beta_{k}\right\}$ in the above argument, we conclude that $n \leqq m$, and the equality follows at once.

In view of Theorem 1 we are justified in speaking of a functional of degree $n$ without reference to any specific real C. O. N. set $\left\{\alpha_{k}(t)\right\}$. More generally if $F(x)$ is a functional of degree $n$ for some unspecified integer $n$, we may speak of $F(x)$ as a functional of finite degree.

Examination of the proof of Theorem 1 enables us to draw another useful conclusion, which we state as a corollary.

COROLlaRY. If $F(x)$ is a (finite) linear combination of functionals of the form $\prod_{j=1}^{g}\left[\int_{0}^{1} f_{j}(t) \tilde{d} x(t)\right]$ where $q \leqq n$, then it is a functional of finite degree; in fact, the degree of $F(x)$ does not exceed $n$.

The next theorem is of some intrinsic interest and will be very useful in the paper on multiple stochastic integrals to which reference was made in the introduction to this paper.

TheOREM 2. Let $F(x)=$ L.I.M.M ${ }_{M \rightarrow \infty} F_{M}(x)$ where, for each $M, F_{M}(x)$ is a functional of degree less than or equal to $n$. Then also $F(x)$ is a functional of degree less than or equal to $n$.

Proof. By mean convergence, 


$$
A_{m_{1}, \cdots, m_{N}}=\lim _{M \rightarrow \infty} \int_{C}^{w} F_{M}(x) \Psi_{m_{1}}, \cdots, m_{N}(x) d_{w} x,
$$

and, by hypothesis, if $m_{1}+\cdots+m_{N}>n$, the integral on the right in (2.6) is zero for each $M$.

3. Convergence of Fourier-Hermite developments. If $F(x) \in L_{2}(C)$, it may be developed in a mean convergent series of Fourier-Hermite polynomials,

$$
F(x)=\underset{N \rightarrow \infty}{\text { L.I.M. }} \sum_{m_{1}, \cdots, m_{N}=0}^{N} A_{m_{1}, \cdots, m_{N}} \Psi_{m_{1}}, \cdots, m_{N}(x) .
$$

If $n$ is any positive integer, we define $S_{N}(x)$ by

$$
S_{N}(x)=\sum_{m_{1}+\cdots+m_{N}=n} A_{m_{1}, \cdots, m_{N}} \Psi_{m_{1}, \cdots, m_{N}}(x) .
$$

Then $\left\{S_{N}(x)\right\}$ converges in the $L_{2}(C)$ mean, so that we may define a functional $S(x) \in L_{2}(C)$ by the equation

$$
S(x)=\underset{N \rightarrow \infty}{\operatorname{LI} . \mathrm{M}} . S_{N}(x) \text {. }
$$

Lastly, for any positive integers $N$ and $K$ and for any positive number $\epsilon$ we define a set $E_{N, \boldsymbol{K}, \mathrm{e}}$ by

$$
E_{N, \mathrm{~K}, \epsilon}=\left\{x: \max _{k \leqq K}\left|S_{N+k}(x)-S_{N}(x)\right|>\epsilon\right\} .
$$

We are now in a position to establish an important preliminary inequality.

LEMMA $2 .^{1} m_{w}\left(E_{N, K, e}\right) \leqq \epsilon^{-2}\left\|S_{N+K}-S_{N}\right\|^{2}$.

Proof. For brevity we write $E=E_{N, K, .0}$ Define $E_{k}$ by

$$
E_{k}=\left\{x: \max _{j<\varepsilon}\left|S_{N+j}(x)-S_{N}(x)\right| \leqq \epsilon,\left|S_{N+k}(x)-S_{N}(x)\right|>\epsilon\right\}
$$

$(1 \leqq k \leqq K)$.

The sets $E_{k}$ are disjoint and $E=\bigcup_{k=1}^{R} E_{k}$. We have

$$
\begin{aligned}
\left\|S_{N+K}-S_{N}\right\|^{2} & \geqq \int_{E}^{w}\left|S_{N+K}(x)-S_{N}(x)\right|^{2} d_{w 0} x \\
& =\sum_{k=1}^{K} \int_{E_{k}}^{\infty}\left|S_{N+K}(x)-S_{N}(x)\right|^{2} d_{w} x=\sum_{k=1}^{K} J_{k} .
\end{aligned}
$$

1 This lemma is an extension of the celebrated Kolmogoroff inequality [2] to the type of problem which concerns us here. 
We have also

$$
\begin{aligned}
J_{k}= & \int_{E_{k}}^{w}\left|S_{N+k}(x)-S_{N}(x)\right|^{2} d_{w} x+2 \operatorname{Re} \int_{E_{k}}^{w}\left[S_{N+k}(x)-S_{N}(x)\right] \\
& \cdot\left[\overline{S_{N+K}(x)-S_{N+k}(x)}\right] d_{w} x+\int_{E_{k}}^{w}\left|S_{N+K}(x)-S_{N+k}(x)\right|^{2} d_{w} x .
\end{aligned}
$$

The second integral on the right-hand side of (3.2) is a finite linear combination of terms of the form

$$
\begin{aligned}
& \int_{E_{k}}^{w}\left\{\prod_{i=1}^{N+k} H_{m_{i}}\left[\int_{0}^{1} \alpha_{i}(t) \tilde{d} x(t)\right]\right\} \\
& \cdot\left\{\prod_{j=1}^{N+K} H_{\mu_{j}}\left[\int_{0}^{1} \alpha_{j}(t) \tilde{d} x(t)\right]\right\} d_{w} x
\end{aligned}
$$

where for at least one $j$, say $j_{0}$, we have

$$
\mu_{j_{0}} \neq 0 \text { and } N+k<j_{0} \leqq N+K \text {. }
$$

As the random variables $X_{\nu}=\int_{0}^{1} \alpha_{\nu}(t) \tilde{d} x(t)$ are statistically independent [3] and as $E_{k}$ is determined wholly by the values of the

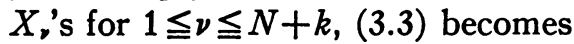

$$
\int_{E_{k}}^{w} G(x) d_{w} x \cdot \int_{C}^{w} H_{\mu_{j_{0}}}\left[\int_{0}^{1} \alpha_{j_{0}}(t) \tilde{d} x(t)\right] d_{w} x,
$$

where $G(x)$ is a certain polynomial in $\int_{0}^{1} \alpha_{\nu}(t) \tilde{d} x(t)$ for $1 \leqq \nu \leqq N+K$ and where $\nu \neq j_{0}$. As $\mu_{j_{0}} \neq 0$, the value of the right-hand integral in (3.4) is zero. It follows that the second integral on the right-hand side of (3.2) vanishes, so that we certainly have

$$
J_{k} \geqq \int_{E_{k}}^{w}\left|S_{N+k}(x)-S_{N}(x)\right|^{2} d_{w} x \geqq \epsilon^{2} m_{w}\left(E_{k}\right) .
$$

From (3.1) and (3.5) we obtain at once

$$
\left\|S_{N+K}-S_{N}\right\|^{2} \geqq \epsilon^{2} \sum_{k=1}^{K} m_{v}\left(E_{k}\right)=\epsilon^{2} m_{w}(E),
$$

from which the assertion of the lemma follows.

It is now a simple matter to establish the convergence theorem mentioned in the introduction.

THEOREM 3. The Fourier-Hermite expansion of a functional $F(x)$ of finite degree converges almost everywhere on $C$. More explicitly, with the notation of the introduction, we have 


$$
\lim _{N \rightarrow \infty} \sum_{m_{1}, \cdots, m_{N}=0}^{N} A_{m_{1}, \cdots, m_{N}} \Psi_{m_{1}}, \cdots, m_{N}(x)=F(x)
$$

for almost all $x \in C$.

Proof. It is obviously sufficient to prove the theorem for the case where the F. H. expansion of $F(x)$ contains only terms of degree $n$. With this understanding and with the notation introduced at the beginning of this section, we have $F(x)=S(x)$ a. e. on $C$. For any $\epsilon>0$ and for any positive integer $N$, define the set $E_{N, \epsilon}$ by the equation

$$
E_{N, \epsilon}=\left\{x: \sup _{k \geqq 1}\left|S_{N+k}(x)-S_{N}(x)\right|>\epsilon\right\} .
$$

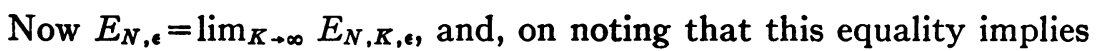
that $\lim _{K \rightarrow \infty} m_{v}\left(E_{N, K, e}\right)=m_{v}\left(E_{N, e}\right)$, we conclude at once by mean convergence and Lemma 2 that

$$
m_{w}\left(E_{N, \epsilon}\right) \leqq \epsilon^{-2}\left\|S-S_{N}\right\|^{2} .
$$

Since $\left\|S-S_{N}\right\| \rightarrow 0,(3.6)$ shows by use of a standard argument that the sequence $\left\{S_{N}(x)\right\}$ converges almost uniformly, and hence almost everywhere on $C$. This remark establishes the theorem.

4. Conclusion. The use of the statistical independence of the functionals $X_{v}=\int_{0}^{1} \alpha_{v}(t) \tilde{d} x(t)$ at the crucial point in the proof of Lemma 2 is worthy of note, as it illustrates the utility of the methods and concepts of mathematical probability in the investigation of an analytical problem. Such statistical arguments will appear again in a critical lemma in the paper on multiple stochastic integrals to which reference was made in the introduction. We take this occasion to point out that the occurrence of statistical arguments at certain crucial points is more or less to be expected in the light of the fact that the Wiener theory is a special case of the general theory of stochastic processes.

\section{BibliograPhy}

1. R. H. Cameron and W. T. Martin, The orthogonal development of non-linear functionals in series of Fourier-Hermite functionals, Ann. of Math. vol. 48 (1947) pp. 385-392.

2. A. Kolmogoroff, Über die Summen durch den Zufall bestimmter unabhängiger Grossen, Math. Ann. vol. 99 (1928) pp. 309-319.

3. R. E. A. C. Paley, N. Wiener, and A. Zygmund, Notes on random functions, Math. Zeit. vol. 37 (1933) pp. 647-668.

Goodyear Aircraft Corporation, Litchfield Park, Arizona 